\title{
Standards and Challenges of Care for Colorectal Cancer Today
}

\author{
Jens Wernera ${ }^{\text {Volker Heinemann }}{ }^{\mathrm{b}, \mathrm{c}}$ \\ ${ }^{a}$ Department of General, Visceral, Transplantation Surgery, Klinikum der Universität München, Ludwig-Maximilians-Universität (LMU), \\ Munich, Germany;

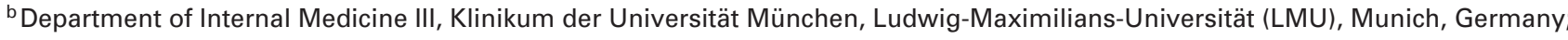 \\ ${ }^{c}$ Comprehensive Cancer Center Munich, Klinikum der Universität München, Ludwig-Maximilians-Universität (LMU), Munich, Germany
}

New diagnostic and therapeutic options for colorectal cancer patients are associated with an increasing complexity of cancer care. Since colorectal cancer is one of the most frequent cancers in the Western world, evidence-based guidelines advocate multimodal treatment regimens as well as the need for interdisciplinary teams. The cornerstones of therapy are surgery, neoadjuvant radio(chemo)therapy (for patients with rectal cancer), and adjuvant chemotherapy. Consequently, centralization of care, certification of oncological centers with quality-assured tumor boards, and establishment of comprehensive cancer centers have led to an increased quality of care as well as to an improved long-term survival throughout Germany. The articles of this special issue focus on different aspects of care for colorectal cancer and describe the standards as well as the latest developments.

Apart from age and hereditary forms, lifestyle factors such as low physical activity, excessive alcohol consumption, smoking, and nutrition are well-known risk factors for colorectal cancer. The latest evidence demonstrates that physical activity may not only reduce the risk of colon cancer but may also be a valuable element of supportive care once a cancer has been diagnosed.

Screening programs are useful and, apart from colonoscopy, tests for occult fecal blood are established. New immunochemical tests have been developed and virtual colonoscopy and capsule endoscopy are promising, though not yet established alternatives to endoscopy. Proper staging of tumor disease is an important prerequisite of optimal subsequent therapy and includes computed tomography, magnetic resonance imaging, and endosonography.

Surgical resection clearly is an important requirement for longterm survival and cure of colorectal cancer. Great improvements of surgical technique, training of surgeons, as well as centralization have increased the quality of resections and long-term survival. Oncological lymph node dissection is required. Thus, total mesorectal excision and the Holms procedure are well-recognized standards for resection of rectal cancer. Other fields of progress include surgery for local recurrence, hepatic and pulmonary metastases as well as peritoneal carcinomatosis. The latter can now successfully be treated by cytoreduction and hyperthermic intraperitoneal chemotherapy in selected cases. While advanced tumor stages are treated more radically today, minimally invasive surgical techniques are also used more and more frequently. They are as reliable and good as open conventional surgical techniques; however, even today only a minority of cases is resected laparoscopically in Germany.

Local resections of low-risk tumors of the rectum can be successfully performed by transanal endoscopic microsurgical techniques without a compromise of oncological outcome. Several minimally invasive methods do exist, but apart from laparoscopy, none is widely used.

For stage II/III rectal cancer, neoadjuvant radiotherapy is superior to adjuvant treatment. While short-course radiation and longcourse chemoradiotherapy (LC-CRT) both reduce the rate of local recurrence, LC-CRT additionally aims to downsize the tumor. Clinical studies presently investigate the optimal choice, duration, and sequence of chemotherapy to be used in combination with radiation.

While surgical resection remains the gold standard for the treatment of liver metastases, several options are available for treating non-resectable metastases. These include radiofrequency ablation, microwave ablation, transarterial chemoembolization, selective internal radiation therapy, and stereotactic body radiotherapy. Multidisciplinary tumor boards are an essential platform for proper discussion of complex cases in the light of multiple treatment options to allow individualized decision making.

Patients with stage III and high-risk stage II colon cancers typically receive adjuvant chemotherapy to reduce the rate of recurrence and allow for improved survival. In stage IV disease, the implementation of targeted therapy has substantially improved the

\section{KARGER}

Fax +497614520714 (c) 2016 S. Karger GmbH, Freiburg

$2297-4725 / 16 / 0323-0156 \$ 39.50 / 0$
Prof. Dr. med. Jens Werner

Klinik für Allgemein-, Viszeral-, Gefäß- und Transplantationschirurgie

LMU Klinikum der Universität München, Campus Großhadern

Marchioninistraße 15, 81377 München, Germany

Jens.Werner@med.uni-muenchen.de 
overall survival to more than 30 months in recent trials. Today, molecular characterization of the tumor including RAS, BRAF, and MSI is a first and important step in the development of a personalized treatment approach. In the molecular subgroups, the selection of proper treatment sequences remains the focus of scientific discussion. New strategies involve the use of anti-PD1(PD-L1)-directed immunotherapies. Despite these advances, life expectancy is still limited in metastatic disease. Therefore, new strategies based on molecular biomarkers are needed. Her2/neu and checkpoint inhibition might be useful in subgroups of patients.

Treatment of colorectal cancer is getting more and more complex. Several medical disciplines are involved and the multimodal treatment approaches need to be discussed in interdisciplinary teams to design the best treatment for the individual patient. Accordingly, the review articles in this issue of Visceral Medicine present today's standards as well as the latest improvements. 